\title{
A Review of Parathyroid Surgery for Primary Hyperparathyroidism from the United Kingdom Registry of Endocrine and Thyroid Surgery (UKRETS)
}

\author{
H. Ishii ${ }^{1}$ (1) - M. J. Stechman ${ }^{2}$ J. C. Watkinson ${ }^{3}$ S. Aspinall ${ }^{4}$ D. S. Kim ${ }^{1}$
}

Accepted: 14 November 2020/Published online: 2 December 2020

(C) The Author(s) 2020

\begin{abstract}
Background The United Kingdom Registry of Endocrine and Thyroid Surgeons is a national database holding details on $>28,000$ parathyroidectomies.

Methods An extract (2004-2017) of the database was analysed to investigate the reported efficacy, safety and use of intra-operative surgical adjuncts in targeted parathyroidectomy (tPTx) and bilateral neck exploration (BNE) for adult, first-time primary hyperparathyroidism (PHPT).

Results $50.9 \%$ of 21,738 cases underwent tPTx. Excellent short-term (median follow-up 35 days) post-operative normocalcaemia rates were reported overall (tPTx 96.6\%, BNE 94.5\%, $p<0.05$ ) and in image-positive cases (tPTx 96.7\%, BNE 96\%, $p<0.05$ ). Intra-operative PTH improved overall normocalcaemia rates (tPTx $97.8 \%$ vs $96.3 \%$, BNE $95 \%$ vs $94.4 \%$ : both $p<0.05$ ). Intra-operative nerve monitoring reduced vocal cord (VC) dysfunction in image-positive tPTx, but not in BNE (97.8\% vs $93.2 \%, p<0.05)$. Complications were higher following BNE $(7.4 \%$ vs $3.8 \%, p<0.05)$, especially hypocalcaemia $(5.3 \%$ vs $2 \%, p<0.05)$. There was no difference in rates of subjective dysphonia following tPTx or BNE (2.4\% vs $2.3 \%, p>0.05)$, nor any difference in VC dysfunction when formally examined ( $4.9 \%$ vs $4.1 \%, p>0.05)$.

Conclusions In image-positive, first time, adult PHPT cases, tPTx is as safe and effective as BNE, with both achieving excellent short-term results with minimal complications.
\end{abstract}

H. Ishii

hiro.ishii.85@gmail.com

1 Department of ENT, Head \& Neck Surgery, St George's Hospital, Blackshaw Road, Tooting, London SW17 0QT, UK

2 Department of Endocrine Surgery, University Hospital Wales, Cardiff, UK

3 Department of Paediatric Surgery, Great Ormond Street Hospital, London, UK

4 Department of General Surgery, Aberdeen Royal Infirmary, Aberdeen, UK

\section{Introduction}

The United Kingdom Registry of Endocrine and Thyroid Surgery (UKRETS) is a multi-centre, multi-disciplinary (General, Otolaryngology, Oral and Maxillofacial, Transplant and Vascular surgeons) database. It is the world's largest endocrine surgery database and is maintained by the British Association of Endocrine and Thyroid Surgeons (BAETS) members.

UKRETS has recognised potential deficiencies in care, including under-diagnosis and heterogeneous practise across the United Kingdom (UK). Therefore, the National Institute for Health and Care Excellence (NICE) have published guidelines on the management of primary hyperparathyroidism (PHPT) in the UK [1]. 
Targeted parathyroidectomy (tPTx) in selected patients, with pre-operative localisation and intra-operative parathyroid hormone (ioPTH), have led to well-documented reductions in operating time and post-operative complications (especially hypocalcaemia) [2-5]. However, there is the potential to miss multiglandular disease (MGD) $[6,7]$, leading to recurrences. ioPTH during tPTx may mitigate this risk, but its benefit in first-time parathyroidectomy remains debatable due to its cost, only moderately high specificity in identifying those cases with remaining abnormal tissue following adenoma excision [8], and marginal improvement in cure [5]. A literature review by Morris et al. [9] demonstrated that in a case of localised PHPT, ioPTH increased the success rate of tPTx from 96.3 to $98.8 \%$, whilst incurring an approximate additional cost of $4 \%$ to the procedure. However, the authors did also conclude that there were institution specific factors that influenced the value of ioPTH.

With this in mind, a review of parathyroid surgery recorded in the UKRETS was undertaken.

\section{Aims}

This study investigated the recorded efficacy and safety of tPTx and bilateral neck exploration (BNE) with and without surgical adjuncts in first-time PHPT cases from the UKRETS.

\section{Material and methods}

Access to UKRETS was granted through the BAETS executive committee in February 2018, for data from 2004 to 2017. Patient consent is obtained by surgeons to collect and anonymously analyse data in UKRETS along General Data Protection Regulation requirements. Clinical data are uploaded by surgeons, but the dataset has not been externally validated. The data fields for parathyroid surgery are available in the appendix of the Fifth National Report [9]. The lead author (HI) analysed the database.

Adults ( $\geq 18$ years old) undergoing first-time parathyroidectomy for sporadic PHPT between 2004 and 2017 were included. Cases with illogical/inaccurate data (e.g. age $>100$ years old) and cases not specified as first-time surgeries were excluded. Cases with incomplete outcome data were excluded from specific sub-analyses to prevent skewing of interpretation. Missing data rates varied across outcomes, so the denominator (total number of cases analysed) varied depending on the outcome in question.

The recorded surgical technique (tPTx or BNE), adjuncts and number of glands removed were analysed. tPTx cases converted to BNE were analysed as BNE.
Complication rates and their nature were analysed. The method (US, indirect or direct laryngoscopy) by which formal vocal cord (VC) function was examined is not specified in UKRETS.

UKRETS does not record operating centre or operative findings, therefore, it was not possible to explain why (failure to identify adenoma, identifying two ipsilateral adenomas, or failure of ioPTH to fall below $50 \%$ of baseline) some tPTx cases were converted to BNE. Neither does it record decision-making on ioPTH or ioNM use, availability of ioPTH/ioNM or operative approach (tPTx or upfront BNE). Therefore, no comments or inferences on these topics were made.

\section{Definitions}

BAETS [8] defines tPTx as "surgery that may include mini-incision open techniques, endoscopic methods or even unilateral neck exploration (UNE)", but UKRETS does not distinguish between these techniques.

"Image-positive" cases where pre-operative localisation modalities (ultrasonography (US) and/or nuclear medicine (NM) and/or computed tomography (CT)/magnetic resonance imaging (MRI)) identified target gland(s). UKRETS does not record of the number or location(s) of the target gland(s).

"Hypocalcaemia" low serum corrected calcium $<2.1 \mathrm{mmol} / \mathrm{L}$ or ionized serum calcium $<1.2 \mathrm{mmol} / \mathrm{L}$ at first post-operative day.

"Normocalcaemia" was defined as the absence of hypocalcaemia and persistent hypercalcaemia at the first post-operative visit.

"Subjective dysphonia" the subjective (assessed by the surgeon) presence of voice change at the first post-operative visit.

\section{Statistical analysis}

Descriptive statistics are reported as medians with interquartile ranges (IQR; 25-75\%), unless stated otherwise. Medians were used to avoid central tendencies being affected by outlying values.

Differences between groups and categorical variables were calculated with the Mann-Whitney U and Fisher's exact test, respectively. $P$-values of $<0.05$ were considered statistically significant.

GraphPad Prism, version 8.0.1 (GraphPad Software, 7825 Fay Avenue, Suite 230, La Jolia, CA 92,037, USA) was utilised for statistical analyses and Microsoft Excel, version 16.12 was used for data handling. 


\section{Results}

After applying exclusions, there were 21,738 first-time PHPT cases between 2004 and 2017 (Fig. 1); 50.9\% $(n=11,062)$ underwent tPTx and $49.1 \% \quad(n=10,676)$ BNE. $8.1 \%(867 / 10,676)$ were converted from tPTx to BNE. Between 2004-07, BNE was most frequently utilised, but since 2008, both techniques have been employed equally (Fig. 2).

BNE and tPTx were performed in 52.2\% (5570/10,676) and $98.1 \%$ of image-positive cases $(10,850 / 11,062)$, respectively. It was not possible to analyse how many of the converted cases were due to a failure of ioPTH levels to drop, as UKRETS did record this. Overall, ioPTH was used more frequently during tPTx $(25 \% ; 63 / 11,602)$ than BNE $(19.1 \%, 2044 / 10,676)(p<0.05)$. ioPTH use has risen from $0 \%(0 / 20)$ of tPTx and 1.3\% (1/77) of BNE in 2004 to $29.8 \%(354 / 1187)$ and $28.6 \%(317 / 1098)$ in 2017, respectively.

On average, 1.25 glands were removed in image-positive BNEs, compared to 1.07 in image-positive tPTx and 1.34 glands in all (image-positive and -negative) BNEs (Table 1).

ioNM use was documented in 3151 cases: $17.5 \%$ of tPTx (1732/9851) versus $15.4 \%$ of BNE cases (1419/9198) $(p<0.05)$. By 2017, ioNM was used in $34.1 \%$ of tPTx (405/1187) and 29.8\% of BNE cases (327/1098). There

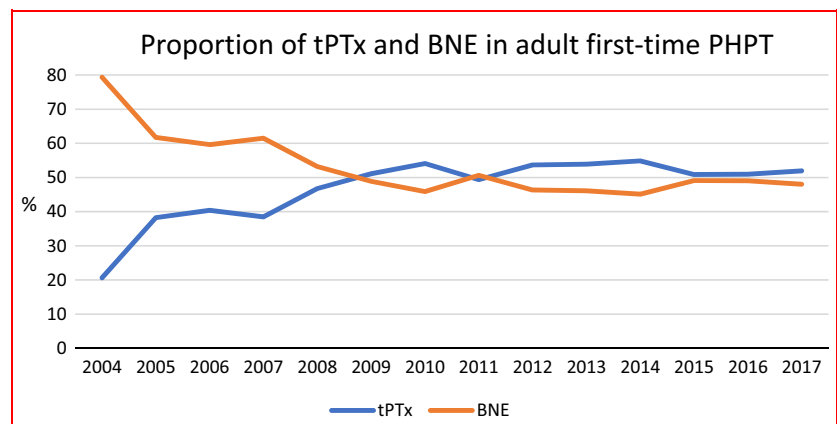

Fig. 2 Proportion of tPTx and BNE in UKRETS between 2004 and 2017 for PHPT

were 1296 parathyroidectomies $(6 \% ; 1296 / 21,738)$ where ioNM use was documented with a formal post-operative VC functional outcome. ioNM was NOT used, but with a formal post-operative VC functional outcome in 2209 parathyroidectomies $(10.2 \%$; 2209/21,738). More cases had normal post-operative VC function following tPTx with ioNM $(97.8 \%$; 657/672), than IPTx without ioNM $(93.2 \% ; 1112 / 1193)(p<0.05)$. There was no difference in VC function with or without ioNM in BNE cases (Table 2). This group represents only $6 \%$ of the dataset due to the infrequent use of ioNM and incomplete datasets.

There was no difference in rates of subjective dysphonia following $\mathrm{tPTx}$ or BNE (2.4\%; $219 / 9003$ vs 2.3\%; $212 / 9093, p>0.05)$. There was no difference between
Fig. 1 Flow diagram of the United Kingdom Registry and Endocrine and Thyroid Surgery database

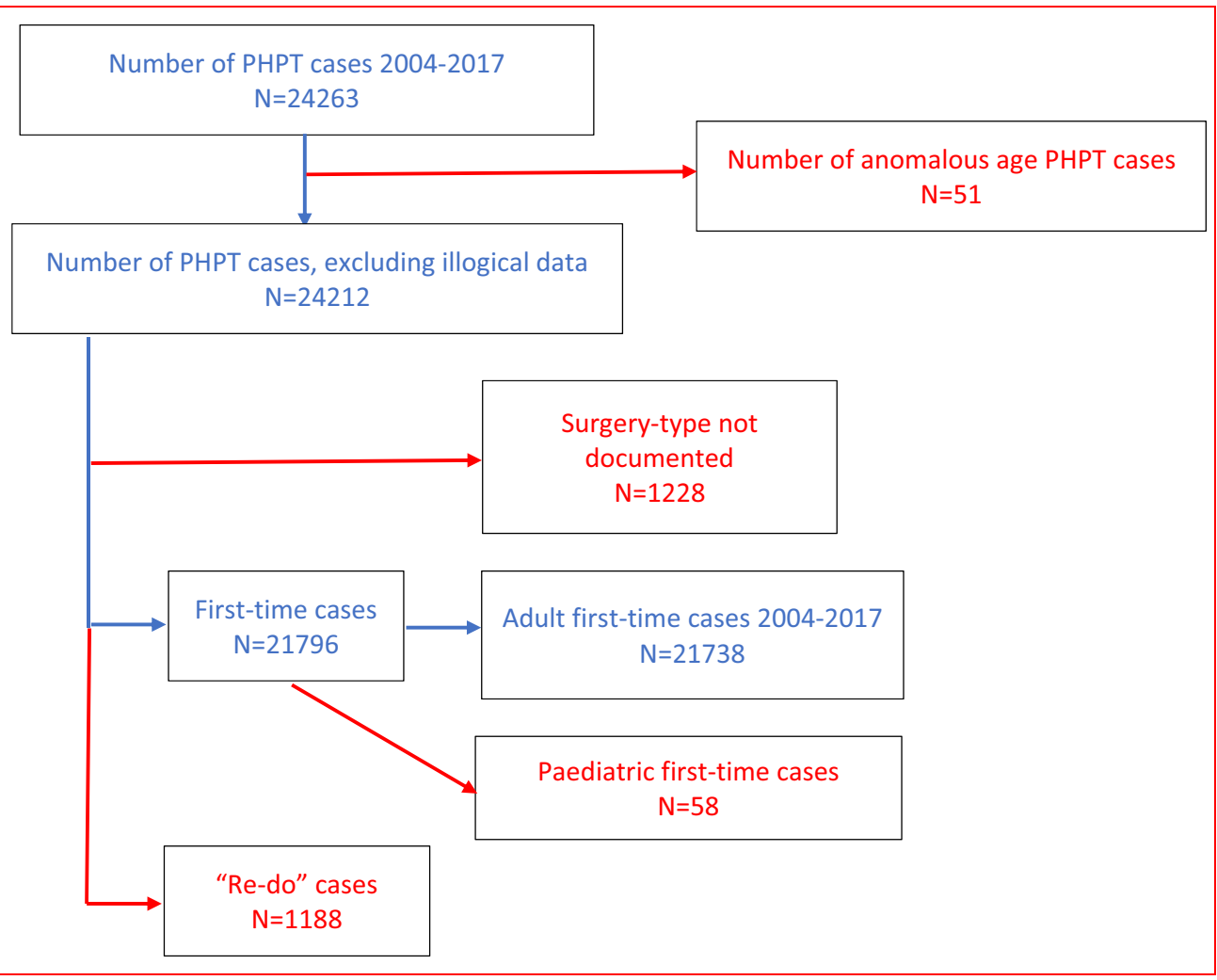


Table 1 Number of parathyroid glands removed per parathyroidectomy

\begin{tabular}{llll}
\hline No. of glands removed & $\begin{array}{l}\text { Image-positive BNE } \\
\text { (total } n=5570)\end{array}$ & $\begin{array}{l}\text { Image negative BNE } \\
\text { (total } n=4029)\end{array}$ & $\begin{array}{l}\text { Image-positive tPTx } \\
(\text { total } n=10,850)\end{array}$ \\
\hline 0 & $166(3.0)$ & $107(2.7)$ & $58(0.5)$ \\
1 & $4043(72.6)$ & $2484(61.7)$ & $9916(91.4)$ \\
2 & $1063(19.1)$ & $871(21.6)$ & $667(6.1)$ \\
3 & $181(3.2)$ & $388(9.6)$ & $70(0.6)$ \\
3.5 & $51(0.9)$ & $93(2.3)$ & $33(0.3)$ \\
4 & $20(0.4)$ & $39(1.0)$ & $11(0.1)$ \\
Total number of glands removed & 6971 & 5872 & 11,620 \\
\hline
\end{tabular}

Values in parentheses are percentages

Table 2 Rates of normal vocal cord movement following tPTx and BNE in UKRETS with and without ioNM

\begin{tabular}{llll}
\hline & With ioNM & Without ioNM & $P$ value \\
\hline Image-positive tPTx & $N(\%)$ & & $1112 / 1193(93.2)$ \\
Image-positive BNE & $657 / 672(97.8)$ & $576 / 604(95.4)$ & $<0.05$ \\
Image negative BNE & $386 / 398(97)$ & $398 / 412(96.7)$ & NS \\
Total no. of cases & $219 / 226(97)$ & $2209 / 21,738(10.2)$ & NS \\
\hline
\end{tabular}

tPTx and BNE in VC dysfunction when formally examined (4.9\%, 93/1890 vs 4.1\%, 70/1716, $p>0.05$ ) (Table 3). Formal VC examination was either not performed or not recorded in most cases $(83.4 \% ; 18,132 / 21,738)$.

The median time to first post-operative visit was 35 days (IQR 19-51). Normocalcaemia rate was higher following tPTx $(96.6 \% ; 8375 / 8664)$ than BNE (94.5\%; 8254/8735) $(p<0.05)$. Normocalcaemia following tPTx was higher with ioPTH $(97.8 \%$; 2291/2343) versus without ioPTH $(96.3 \% ; 6084 / 6321)(p<0.05)$. There was no difference in normocalcaemia rates following BNE with or without ioPTH $(95.0 \%$; $1603 / 1688$ vs $94.4 \%$; 6651/7047, $p>0.05$ ) (Table 4).

Image-positive tPTx had higher normocalcaemia rates than BNE (96.7\%; 8126/8397 vs $96.0 \% ; 4131 / 4305)$ $(p<0.05)$ and especially when ioPTH was used $(97.8 \%$; $2200 / 2249$ vs $96.1 \%$; 730/760) $(p<0.05)$. There was no difference in normocalcaemia rates between image-positive tPTx (96.4\%; 5924/6148) and BNE (96.0\%; 3401/3545) without ioPTH. Normocalcaemia rates following BNE overall were lower than those in image-positive BNE (94.5\%; 6651/7047 vs 96.0\%; 3401/3545) $(p<0.05)$ (Table 4). Missing data represented $17.4 \%$ $(3430 / 21,738)$ of cases.

Overall, BNE had a higher complication rate than tPTx (7.4\%; 786/10,676 vs 3.8\%; 417/11,062) (p <0.05), mostly due to hypocalcaemia $(5.3 \% ; 533 / 10,131$ vs $2.0 \%$; 211/10,360) (Table 5).

Image-positive BNE had higher complication rates than image-positive tPTx (4.9\%; 274/5570 vs 3.5\%; $376 / 10,850)(p<0.05)$. Wound infection and hypocalcaemia rates were lower following image-positive tPTx compared to image-positive BNE (Table 6). Analysis of image-positive cases where only one gland was removed

Table 3 Incidence and missing data for subjective dysphonia and abnormal VC assessment following parathyroidectomy, comparing outcomes from tPTx and BNE

\begin{tabular}{llll}
\hline & tPTx $(\%)$ & BNE $(\%)$ & $P$ value \\
\hline Subjective dysphonia & $219 / 9003(2.4)$ & $212 / 9093(2.3)$ & NS \\
Post-op VC check done & $1890 / 11,062(17.1)$ & $1716 / 10,676(16.1)$ & NS \\
Post-op abnormal VC check & $93 / 1890(4.9)$ & $70 / 1716(4.1)$ & NS \\
\hline
\end{tabular}


Table 4 Rate of normocalcaemia following parathyroidectomy comparing tPTx and BNE with and without ioPTH in overall and image-positive cases

\begin{tabular}{llll}
\hline & tPTx $(\%)$ & BNE $(\%)$ & $P$ value \\
\hline Overall & $8375 / 8664(96.6)$ & $8254 / 8735(94.5)$ & $<0.05$ \\
With ioPTH & $2291 / 2343(97.8)$ & $1603 / 1688(95.0)$ & $<0.05$ \\
Without ioPTH & $6084 / 6321(96.3)$ & $6651 / 7047(94.4)$ & $<0.05$ \\
\hline & Image + ve tPTx $(\%)$ & Image + ve BNE $(\%)$ & $<0.05$ \\
\hline Overall & $8126 / 8397(96.7)$ & $4131 / 4305(96.0)$ & $<0.05$ \\
With ioPTH & $2200 / 2249(97.8)$ & $730 / 760(96.1)$ & $3401 / 3545(96.0)$ \\
Without ioPTH & $5926 / 6148(96.4)$ & & NS \\
\hline
\end{tabular}

Table 5 Overall complications following tPTx and BNE

\begin{tabular}{llll}
\hline Complications & tPTx $(\%)$ & BNE $(\%)$ & P value \\
\hline Overall complication rate & $417 / 11,062(3.8)$ & $786 / 10,676(7.4)$ & $<0.05$ \\
Wound infection & $28 / 11,062(0.3)$ & $47 / 10,676(0.4)$ & $<0.05$ \\
Haematoma requiring RTT & $36 / 10,505(0.3)$ & $44 / 10,195(0.4)$ & NS \\
Hypocalcaemia (NOS) & $211 / 10,360(2.0)$ & $533 / 10,131(5.3)$ & $<0.05$ \\
Other complications & $142 / 11,062(1.3)$ & $162 / 10,676(1.5)$ & NS \\
\hline
\end{tabular}

$N S$ not significant, $N O S$ not otherwise specified, $R T T$ return to theatre

Table 6 Complications following parathyroidectomy in image positive cases

\begin{tabular}{lllr}
\hline Complications & Image + ve tPTx $(\%)$ & Image + ve BNE $(\%)$ & $P$ value \\
\hline Overall complication rate & $376 / 10,850(3.5)$ & $274 / 5570(4.9)$ & $<0.05$ \\
Wound infection & $23 / 10,850(0.2)$ & $26 / 5570(0.5)$ & $<0.05$ \\
Haematoma requiring RTT & $36 / 10,310(0.3)$ & $14 / 3357(0.4)$ & NS \\
Hypocalcaemia (NOS) & $203 / 10,188(2.0)$ & $165 / 3335(4.9)$ & $<0.05$ \\
Other complications & $114 / 10,850(1.1)$ & $69 / 5570(1.2)$ & NS \\
\hline
\end{tabular}

that resulted in hypocalcaemia demonstrated tPTx had a lower rate that BNE $(2.6 \% ; 159 / 6062$ vs $3.5 \%$; 141/4043, $p<0.05)$.

Of 744 hypocalcaemic cases, 434 (58.3\%) had records of whether or not vitamin D/calcium supplements were still being taken at the first post-operative visit. Fewer tPTx cases were taking supplements compared to BNE cases $(13.1 \% ; 18 / 137$ vs $24.3 \% ; 72 / 296, p<0.05)$. Unfortunately, there is no long-term hypocalcaemia data.

$21.2 \%$ of tPTxs $(2068 / 9838)$ were discharged on the same day, versus $11.2 \%$ of BNEs $(1089 / 9787)(p<0.05)$. There was no difference in discharge frequency on the first post-operative day (tPTx 66.2\%; 6514/9838 vs BNE $66.4 \% ; 6500 / 9787, p>0.05)$. Length of stay greater than one day was more common in BNE than tPTx $(22.5 \%$; $2198 / 9787$ vs $12.8 \%$; $1256 / 9838)(p<0.05)$.

\section{Discussion}

This analysis of 21,738 adult first-time PHPT cases between 2004 and 2017 is the largest reported multi-centre, multidisciplinary review of parathyroid surgery in the world. 11,602 (50.9\%) underwent tPTx, comparable to other registry studies; Kazaure et al. [10] (55.9\%, $n=3592)$ and Bergenfelz et al. [11] (39\%, $n=1050)$.

Despite positive pre-operative localisation, $52 \%$ of patients underwent BNE. $8 \%$ of these $(867 / 10,676)$ were initially treated as $\mathrm{APTx}$ then converted to BNE, inferring 
that $44 \%$ of image-positive cases underwent upfront BNE. It was not possible to report if cases underwent BNE due to multiple glands being identified on pre-operative localisation, which may explain why a high proportion of cases underwent BNE with positive localisation. Other potential hypotheses include surgeon preference on operative technique, variable imaging quality and patient factors (high body mass index) and shared decision-making.

Normocalcaemia rates following both techniques were excellent and are comparative to reports by Jinih et al. [12] (tPTx 96.4\%, BNE 96.7\%; no clear definition of “cure") and Ospina et al. [13] (tPTx 98\%, BNE 97\%; variable definition of "cure"). The authors acknowledge that "normocalcaemia" in this study does not equate to the American Association of Endocrine Surgeons (AAES) definition of "cure" [2]. The UKRETS does not currently collect data on long-term (more than six months) follow-up data. Normocalcaemia rates for image-positive cases suggestive of single gland disease managed by tPTx and BNE (96.7\% vs $96.0 \%$ ), although statistically significant, were marginal and so of questionable clinical importance. This supports the recommendation of discussing both operative approaches in image-positive patients [1].

Normocalcaemia rates following tPTx were excellent with $(97.8 \%)$ or without $(96.4 \%)$ ioPTH. These figures underestimate the benefit of ioPTH, as cases converted from tPTx to BNE, possibly due to failure of ioPTH to fall below $50 \%$ of baseline, were analysed as BNE, and so not included in the analysis of tPTx. Ishii et al. [14] reported overall cure at six months was higher $(99.3 \%$ vs $98.1 \%$, $p<0.05)$ and recurrence lower $(0.2 \%$ vs $1.5 \%, p<0.05)$ when ioPTH was utilised with minimally invasive parathyroidectomy. They found ioPTH was less likely to be utilised in image concordant (versus image discordant) cases. The low record of ioPTH in UKRETS suggests it is still not being routinely used. However, the database demonstrated that the rate of ioPTH was at its highest in 2017. NICE [1] does not recommend routine use of ioPTH in first-time surgery, which seems justified, given the extra cost, for only marginal improvements in normocalcaemia rates. Neither NICE or AAES currently recommend routine use of ioNM [1,2]. UKRETS demonstrated, in a small subset of cases $(6 \%)$ which reported ioNM use with a documented post-operative VC assessment outcome, there were fewer abnormal VC function following tPTx, but not BNE. It is unclear why ioNM should be beneficial in tPTx but not BNE, though limited dissection during tPTx may compromise a satisfactory view of the recurrent laryngeal nerve. ioNM was used more frequently in tPTx than BNE, probably due to increasing popularity of tPTx coinciding with increasing availability of ioNM. Given the small proportion of cases with reported ioNM use, these results may not be representative of or applicable to overall care.
However, UKRETS demonstrated an association between ioNM in TPTx and improved rates of post-operative normal $\mathrm{VC}$ function.

Overall and image-positive complication rates were significantly lower following tPTx versus BNE. These figures are similar to Jinih et al. [12] (tPTx 3.7\%, BNE $17.1 \%$ ). Bergenfelz et al.'s [11] review of the SQRTPA database reported overall complication rates of $3.4 \%$ $(n=92)$.

It was not possible to ascertain if hypocalcaemia was temporary or permanent or if there was a cause such as hungry bone syndrome. Hypocalcaemia rates were significantly higher following BNE than tPTx, but this study found that more glands were excised during BNE, so this higher rate is expected.

Abnormal VC function following tPTx (4.9\%) was higher than Jinih et al.'s [12] and Ishii et al.'s [14] numbers. The high rate of missing data for VC function meant it was difficult to draw any definitive conclusions. Subjective dysphonia rate was $2.4 \%$ (no difference between techniques). However, subjective assessment is inaccurate, variable and may potentially underestimate actual VC dysfunction [15, 16]. Rates of formally assessed post-operative abnormal VC function were $4.9 \%$ following tPTx and $4.1 \%$ following $\mathrm{BNE}(p>0.05)$, but it is not fair to compare these figures against subjective dysphonia rates as there may be other reasons for dysphonia (injury to external branch of superior laryngeal nerve, vocal cord oedema, surgical scar tissue, etc.).

BAETS do not endorse same-day discharge following thyroidectomy due to potential airway obstruction secondary to post-operative haematoma [17]. This has influenced parathyroidectomy practise and may explain the similar proportion of overnight stays following tPTx and BNE. BNEs had longer post-operative stays which may be partially explained by the higher hypocalcaemia rate in this group, where normalising calcium may have required intravenous replacements, delaying discharge.

\section{Strengths and limitations}

Registries are useful to answer certain questions that are not suitable for randomized clinical trials. This registry study provides the best insight into parathyroidectomy outcomes across the UK. Multi-disciplinary surgeons have contributed from both small and large volume centres, reducing the risk of skewed data, and allowing comparison with recognised guidelines [1, 2]. The large number of cases reduces the risk of bias/errors that are inherent from small sample sizes, and highlights statistically significant differences between groups with similar clinical outcomes. It is important to consider these results carefully, as the 
number of patients needed to treat to achieve comparable results may not justify expensive interventions for only marginal benefits.

Limitations include the significant amount of missing data, design flaws of data collection, potential self-reporting bias, coding errors, voluntary nature of uploading cases and lack of external validation (though this is currently being addressed). The results only allowed a median follow-up of 35 days, meaning long-term outcomes (cure, persistent disease and recurrence rates) were not recorded, limiting its use when comparing techniques. "Targeted parathyroidectomy" as per UKRETS, encompasses various surgical techniques, which may affect outcomes. Selection criteria for surgery, symptoms, complications or severity of PHPT are not recorded and therefore not assessed.

An example of potential coding errors is highlighted in the number of glands removed by operative technique. An average of 1.07 glands was excised during tPTx, most likely because UNEs are coded as tPTx in UKRETS. It is not possible to explain why 3, 3.5 or 4 glands were excised during tPTx, as UKRETS does not record pre-operative findings or histology of excised glands, though presumably these may be coding errors.

Factors confounding the interpretation of results include the fact that only selected patients underwent tPTx, therefore the incidence of MGD is likely to be higher in cases undergoing BNE, where more glands were excised. This affects comparisons of normocalcaemia and hypocalcaemia rates between tPTx and BNE. However, this study did demonstrate that in image-positive cases where only one gland was removed, BNE had a higher rate of postoperative hypocalcaemia than tPTx.

Interpretation of localisation results are limited. The number anatomical location of pathological glands or whether the results corresponded to operative findings are not recorded. Hence any inferences about the concordance or discordance of localisation modalities cannot be made.

This study has highlighted areas of the registry that can be updated to improve data collection and analysis.

\section{Conclusions}

This study has reported on the largest parathyroid registry in the world; 21,738 cases of first-time primary hyperparathyroidism treated between 2004 and 2017. This study has demonstrated that in image-positive cases, tPTx is as safe and effective as BNE, with both achieving excellent results with minimal complications.

Acknowledgements The authors would like to thank the BAETS executive committee for allowing access to the database to make this paper possible and the BAETS members listed below who contributed data to the database over this time period.

Richard Adamson, Anna Aertssen, Ahmed Afzaal, Avi Agrawal, Afzaal Ahmad, Ijaz Ahmad, Orabi Ahmad, Ibrahim Ahmed, Irfan Akhtar, Murat Akyol, Peyman Alam, Munther Aldoori, David Allen, Iain D Anderson, Sebastian Aspinall, Chris Ayshford, Ekambaram Dinakara Babu, Christopher Backhouse, Saba Balasubramanian, Alistair Balfour, Neal Banga, Ludger Barthelmes, Nigel Beasley, Chris Bem, Ian Black, Stephen Blair, Richard Bliss, Victoria Brown, Robert Carpenter, Michael Carr, AndreW Carswell, Carmen de Casso Moxo, David Chadwick, Habib Charfare, Andy Chin, Edward Chisholm, Louise Clark, Peter Clarke, Helen Cocks, Peter Conboy, Luke Condon, Rogan Corbridge, Allan Paul Corder, Paul Counter, Stephen P Courtney, Eamonn Coveney, Hugh Cox, Wendy Craig, James N Crinnion, David Cunliffe, Titus Cvasciuc, Jeremy P Davis, Stuart Denholm, Ganapathy Dhanasekar, Vikram Dhar, Ann Dingle, James Docherty, Helen Doran, Julie Dunn, Fiona Eatock, Anusha Edwards, Wael Elsaify, James England, Abigail A Evans, Roy Farrell, Brian Fish, Bence Forgacs, Clare Fowler, Georgios Fragkiadakis, Gabriele Galata, Ashu Gandhi, Richard Garth, Ajith George, Nicholas Gibbins, Martin G Greaney, Thomas G Groot-Wassink, Paul Gurr, Andrew Guy, Wayne Halfpenny, Charles Hall, Paul Hans, Robert Hardy, Churunal Hari, Barney Harrison, Micheal Harron, Simon Hickey, Omar Hilmi, Tim Hoare, Jonathan Hobson, Philip Holland, Andrew Houghton, David Howe, Jonathan Hubbard, Neil Hulton, Paul Hurley, Andrew Husband, Aidah Isa, Shaun Jackson, Tony Jacob, Sharan Chakkyath Jayaram, Jean- Pierre Jeannon, Taleb Jeddy, Stephanie Jenkins, Bethan Jones, Anton Joseph, Bengt Kald, Robert Kennedy, James Kirkby-Bott Paul Kirkland, Ursula Kirkpatrick, Zygmunt Krukowski, Nirmal Kumar, Vijay Kurup, Tom Kurzawinski, Nicholas RF Lagattolla, Mark Lansdown, Nicholas Law, Tom WJ Lennard, Peter Lewis, Andrew P Locker, John RC Logie, Sean Loughran, M Lucarotti, John Lynn, Alasdair Mace, Fiona MacGregor, Paul R Maddox, Arcot Maheshwar, Zvoru Makura, Deborah Markham, Dominic Martin-Hirsch, Andrew Mccombe, Julian McGlashan, Andrew McIrvine, Andrew J McLaren, Sandy McPherson, Hesham Mehanna, Radu Mihai, Faisal MA Mihaimeed, Tedla Miroslav, George Mochloulis, James Moor, Peter Moore, Ram Moorthy, Pradeep Morar, Justin Morgan, Iain M Muir, Michael L Nicholson, Stewart Nicholson, Keshav Nigam, Iain Nixon, Janet O'Connell, Olawale Olarinde, Fausto Palazzo, Michael Papesch, Neil R Parrott, Susannah Penney, Andrew Pfleiderer, Jonathan Philpott, Lisa Pitkin, Isabel Quiroga, David Ratliff, Duraisamy Ravichandran, Venkat Reddy, David Rew, Keith Rigg, Nick Roland, Alasdair Ross, Tom Rourke, Gavin T Royle, Sarwat Sadek, Greg Sadler, Mrinal Saharay, Michael, Ahmed Samy, Klaus- Martin Schulte, David ScottCoombes, Anup Kumar Sharma, Steve Shering, Susannah Shore, John Shotton, Richard Sim, Ricard Simo, Prakash Sinha, Gunasekaran Sinnappa, Anthony Skene, James Smellie, David M Smith, Ian Smith, Simon Smith, Roy Spence, Paul Spraggs, Adam Stacey-Clear, Frank Stafford, Michael P Stearns, Michael Stechman, Paul Stimpson, Robert Sudderick, Robert Sutclffe, Peter Tassone, Taranjit Tatla, Gareth Tervit, Paul Thomas, Adrian Thompson, Steven Thrush, Paul Tierney, Augustine Titus, Neil Tolley, Mark Tomlinson, Paul Turner, Charanjeit S Ubhi, Harpreet Uppal, Srinivasan Venkat, Richard Vowles, Alison Waghorn, Jonathan C Watkinson, Gavin Watters, John Weighill, Andrew R Welch, Hugh Wheatley, Martin Wickham, Chandana Wijewardena, Adam Wild, Michael R Williams, Simon Williams, Paul Wilson, Michail Winkler, Stephen Wood, Constantinos Yiangou, Charles Zammitt.

Funding This research did not receive any specific grant from funding agencies in the public, commercial, or not-for-profit sectors. 


\section{Compliance with ethical standards}

Conflict of interest The authors declare that they have no conflict of interest.

Ethical approval There were no ethical or Institutional Review Board (IRB) considerations applicable to this study.

Open Access This article is licensed under a Creative Commons Attribution 4.0 International License, which permits use, sharing, adaptation, distribution and reproduction in any medium or format, as long as you give appropriate credit to the original author(s) and the source, provide a link to the Creative Commons licence, and indicate if changes were made. The images or other third party material in this article are included in the article's Creative Commons licence, unless indicated otherwise in a credit line to the material. If material is not included in the article's Creative Commons licence and your intended use is not permitted by statutory regulation or exceeds the permitted use, you will need to obtain permission directly from the copyright holder. To view a copy of this licence, visit http://creativecommons. org/licenses/by/4.0/.

\section{References}

1. Overview I Hyperparathyroidism (primary): diagnosis, assessment and initial management I Guidance I NICE. Published 2019. https://www.nice.org.uk/guidance/ng132

2. Wilhelm SM, Wang TS, Ruan DT et al (2016) The American association of endocrine surgeons guidelines for definitive management of primary hyperparathyroidism. JAMA Surg 151(10):959-968. https://doi.org/10.1001/jamasurg.2016.2310

3. Udelsman R, Donovan PI, Sokoll LJ (2000) One hundred consecutive minimally invasive parathyroid explorations. Ann Surg 232(3):331-339

4. Russell CFJ, Dolan SJ, Laird JD (2006) Randomized clinical trial comparing scan-directed unilateral versus bilateral cervical exploration for primary hyperparathyroidism due to solitary adenoma. Br J Surg 93(4):418-421. https://doi.org/10.1002/bjs. 5250

5. Westerdahl J, Bergenfelz A (2007) Unilateral versus bilateral neck exploration for primary hyperparathyroidism: five-year follow-up of a randomized controlled trial. Ann Surg 246(6):971-976. https://doi.org/10.1097/SLA.0b013e31815c3ffd

6. Norman J, Lopez J, Politz D (2012) Abandoning unilateral parathyroidectomy: Why we reversed our position after 15,000 parathyroid operations. J Am Coll Surg 214(3):260-269. https:// doi.org/10.1016/j.jamcollsurg.2011.12.007
7. Hodin R, Angelos P, Carty S et al (2012) No need to abandon unilateral parathyroid surgery. J Am Coll Surg 215(2):297. https://doi.org/10.1016/j.jamcollsurg.2012.04.024

8. Siperstein A, Berber E, Barbosa GF et al (2008) Predicting the success of limited exploration for primary hyperparathyroidism using ultrasound, sestamibi, and intraoperative parathyroid hormone: analysis of 1158 cases. Ann Surg 248(3):420-428. https:// doi.org/10.1097/SLA.0b013e3181859f71

9. Morris LF, Zanocco K, Ituarte PHG et al (2010) The value of intraoperative parathyroid hormone monitoring in localized primary hyperparathyroidism: a cost analysis. Ann Surg Oncol 17(3):679-685. https://doi.org/10.1245/s10434-009-0773-1

10. Kazaure HS, Thomas S, Scheri RP, Stang MT, Roman SA, Sosa JA (2019) The devil is in the details: Assessing treatment and outcomes of 6,795 patients undergoing remedial parathyroidectomy in the collaborative endocrine surgery quality improvement program. Surg (United States) 165(1):242-249. https://doi.org/10. 1016/j.surg.2018.03.026

11. Bergenfelz AOJ, Wallin G, Jansson S et al (2011) Results of surgery for sporadic primary hyperparathyroidism in patients with preoperatively negative sestamibi scintigraphy and ultrasound. Langenbeck's Arch Surg 396(1):83-90. https://doi.org/10. 1007/s00423-010-0724-0

12. Jinih M, O'Connell E, O'Leary DP, Liew A, Redmond HP (2016) Focused Versus Bilateral Parathyroid Exploration for Primary Hyperparathyroidism: A Systematic Review and Meta-analysis. Ann Surg Oncol. https://doi.org/10.1245/s10434-016-5694-1

13. Singh Ospina NM, Rodriguez-Gutierrez R, Maraka S et al (2016) Outcomes of parathyroidectomy in patients with primary hyperparathyroidism: a systematic review and meta-analysis. World J Surg 40(10):2359-2377. https://doi.org/10.1007/s00268-0163514-1

14. Ishii H, Mihai R, Watkinson JC, Kim DS (2018) Systematic review of cure and recurrence rates following minimally invasive parathyroidectomy. BJS Open. https://doi.org/10.1002/bjs5.77

15. Chandrasekhar SS, Randolph GW, Seidman MD et al (2013) Clinical practice guideline: improving voice outcomes after thyroid surgery. Otolaryngol Neck Surg. 148(6_suppl):s1-s37. https://doi.org/10.1177/0194599813487301

16. Bergenfelz A, Jansson S, Kristoffersson A et al (2008) Complications to thyroid surgery: results as reported in a database from a multicenter audit comprising 3,660 patients. Langenbeck's Arch Surg 393(5):667-673. https://doi.org/10.1007/s00423-008-0366-7

17. Doran HE, England J, Palazzo F (2012) British Association of Endocrine and Thyroid Surgeons. Questionable safety of thyroid surgery with same day discharge. Ann R Coll Surg Engl. 94(8):543-547. https://doi.org/10.1308/003588412X1337340538 4576

Publisher's Note Springer Nature remains neutral with regard to jurisdictional claims in published maps and institutional affiliations. 